\title{
Primary splenic leiomyosarcoma - case report and literature review
}

\author{
Elena Simona Ioanițescu ${ }^{1,2}$, Mugur Grasu ${ }^{2,3}$, Letiția Toma ${ }^{1,2}$
}

${ }^{1}$ Department of Internal Medicine, Fundeni Clinical Institute, ${ }^{2}$ "Carol Davila" University of Medicine and Pharmacy, ${ }^{3}$ Department of Interventional Radiology, Fundeni Clinical Institute, Bucharest, Romania

\begin{abstract}
Primary tumors of the spleen are rarely encountered in clinical practice and their diagnosis often requires invasive procedures (splenectomy). Leiomyosarcomas are rare tumors originating from smooth muscle cells or their precursor mesenchymal cells and as such can arise in any organs, most typically abdominal ones. Only a few cases of leiomyosarcomas of the spleen have been described in literature. We present the case of a 69 year-old, a previously healthy patient, with non-specific symptoms, diagnosed on CT scan with multiple splenic, hepatic and bone tumors. Biopsy from one of the liver tumors revealed the diagnosis of leiomyosarcoma. Due to characteristic aspects on contrast-enhanced ultrasonography and CT scan we concluded that the primary tumor was located in the spleen, while the others represented metastases.

Keywords: leiomyosarcoma; spleen; contrast-enhanced ultrasonography
\end{abstract}

\section{Introduction}

Malignant tumors of the spleen can be classified as lymphoid, non-lymphoid and metastases, originating most frequently from melanomas, breast and lung cancers [1]. Primary involvement of the spleen in lymphomas is much rarer than splenic infiltration during the course of the disease [2] and the most frequently encountered are non-Hodgkin's lymphomas originating in B cells [3]. Non-lymphoid malignant tumors are rare and include sarcomas, angiosarcomas or malignant teratomas [1].

A clear diagnosis regarding focal splenic lesions is difficult to achieve by imaging means, particularly due to imaging similarities and the scarcity of particular lesions [4-6]. Ultrasonography is the first step in diagnosis and evaluation of splenic tumors, and contrast-enhanced ultrasonography (CEUS) can add important information regarding the characteristics of focal splenic lesions [7].

Received 22.06.2021 Accepted 11.09.2021

Med Ultrason

2022, Vol. 24, No 1, 114-116

Corresponding author: Elena Simona Ioanițescu

Department of Internal Medicine,

Fundeni Clinical Institute, 258 Fundeni Street,

Bucharest, Romania, 022328

E-mail simona.ioanitescu@gmail.com

Phone: +40722494586
Leiomyosarcomas are rare tumors originating in smooth muscle cells; therefore, they may affect any organ with a predilection for abdominal organs and the uterus. They are so rare that they have not been included in the latest WHO classification of splenic tumors [8]. To our knowledge, there have been only three cases of primary leiomyosarcomas of the spleen described in literature.

\section{Case report}

A 69 year-old female patient was admitted for diffuse abdominal pain and general malaise, progressively accentuated over the past two weeks. The patient had no previously diagnosed conditions and had no chronic medication. She denied constipation or diarrhea, as well as melena or hematochezia. She was a non-smoker and had no history of alcohol or illicit drug use. On admission, the patient signed an informed consent for scientific and research purposes.

Clinical evaluation revealed a pale, dehydrated patient, with sclera jaundice, oxygen saturation of $97 \%$ in ambient air, blood pressure $99 / 69 \mathrm{mmHg}$ and 56 beats/ minute heart rate, bilateral lower limb edema. Abdominal exam revealed a diffusely tender abdomen, with enlarged liver ( $5 \mathrm{~cm}$ below lower rib) and spleen $(4 \mathrm{~cm}$ below lower rib); there were no signs of acute abdomen. Neurologic 

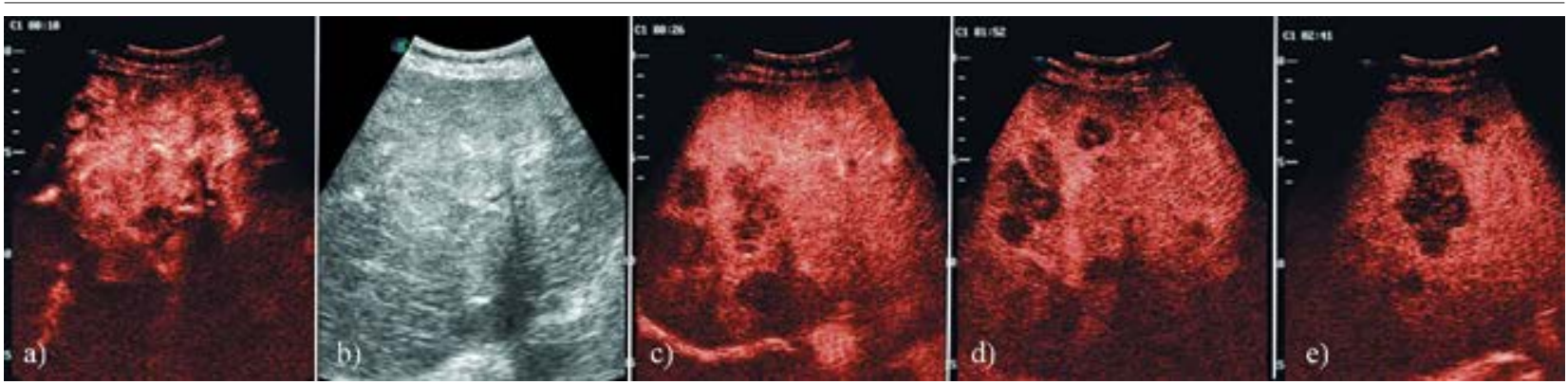

Fig 1. Liver CEUS examination: a) and c) arterial phase revealed iso-hypoenhancement followed by early wash out; d) and e) portal and late phase showing progressive and marked wash out; b) reference image.
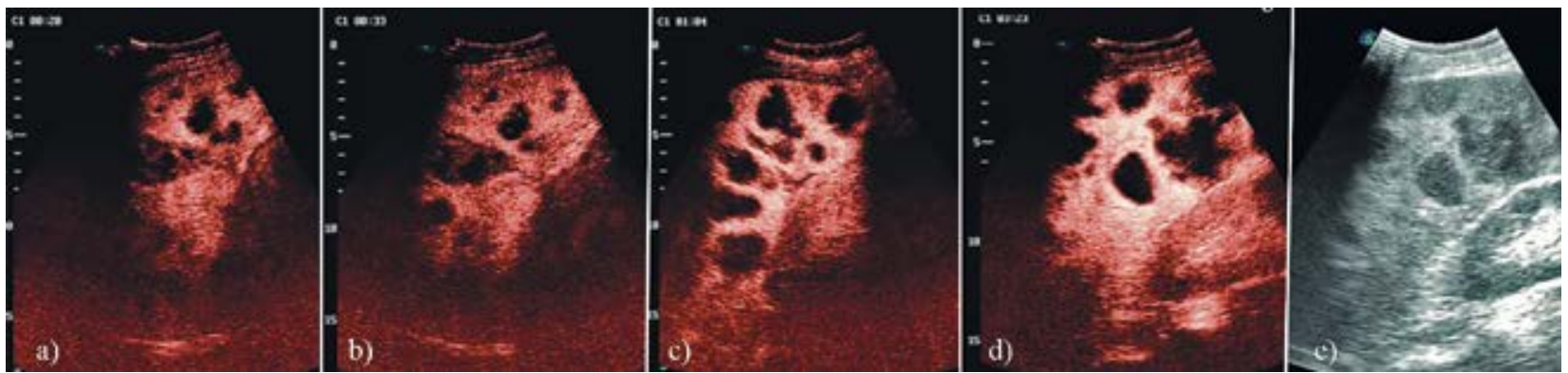

Fig 2. CEUS examination of the spleen: a) and b) arterial phase revealed multiple cystic lesions with slightly hypoenhancement of septae and walls in some lesions, the majority isoenhancing; c) and d) venous and late phase; e) reference image.

evaluation revealed a disoriented patient, without other neurologic deficits.

Blood tests were suggestive for liver insufficiency (hypoalbuminemia, hypocholesterolemia) as well as signs of kidney injury (creatinine $2.02 \mathrm{mg} / \mathrm{dL}$ ), increased levels of uric acid $(11.7 \mathrm{mg} / \mathrm{dL})$, severe anemia $(6.6 \mathrm{~g} /$ dL) and thrombocytopenia. Serum markers for chronic viral hepatitis as well as for autoimmune disorders of the liver were negative and the patient did not have proteinuria. The patient was negative for HIV.

Abdominal ultrasonography revealed moderate ascites, enlarged lymph nodes in the upper abdomen, enlarged liver with multiple hypoechoic tumors, measuring up to $5 \mathrm{~cm}$, important splenomegaly, with a long axis of 21 $\mathrm{cm}$, with multiple focal lesions apparently similar to those from the liver. CEUS was performed revealing arterial iso-hypoenhacement of the liver lesions with rapid washout, highly suggestive for liver metastases (fig 1). CEUS of the spleen showed multiple cystic lesions, some of them with septae, located in almost the entire spleen. The lesions present arterial peripheral rim enhancement with slightly or no wash-out (fig 2). Upper abdominal lymph nodes were non-enhancing, suggesting necrosis (fig 3).

Upper endoscopy and colonoscopy had no pathologic findings. Thoraco-abdominal CT scan revealed bilateral pleural fluid, enlarged mediastinum, abdominal lymph nodes and multiple hypodense liver and splenic lesions, the largest located at the inferior splenic pole $(93 \mathrm{~mm})$ (fig 4). A lytic lesion to the L1 vertebral body was also revealed on CT-scan. Due to accessibility, we performed a CT-guided biopsy of the liver lesions. Histopathological analysis showed a mesenchymal proliferation of fusiform and epithelioid cells. Immune-histochemical test were negative for CD117, CD34, WT1, DOG1 (excluding gastro-intestinal stromal tumor), and positive for Vimentin (confirming mesenchymal origin) and SMA (specific for myofibroblasts).

We concluded that the diagnosis was primary leiomyosarcoma of the spleen associated with liver, bones and lymph nodes metastases. Due to the poor clinical status of the patient, as well as the extension of disease, the patient was referred for palliative treatment.

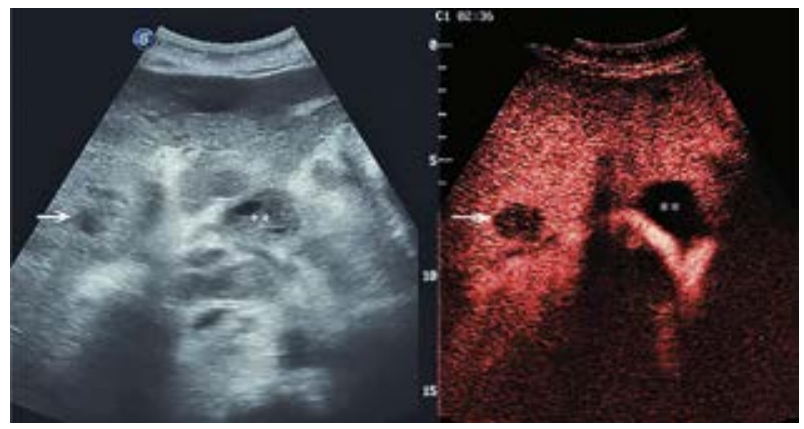

Fig 3. CEUS examination, late phase. Non-enhancing celiac lymph node, suggesting necrosis (asterisc). Arrow indicates a liver metastasis. 


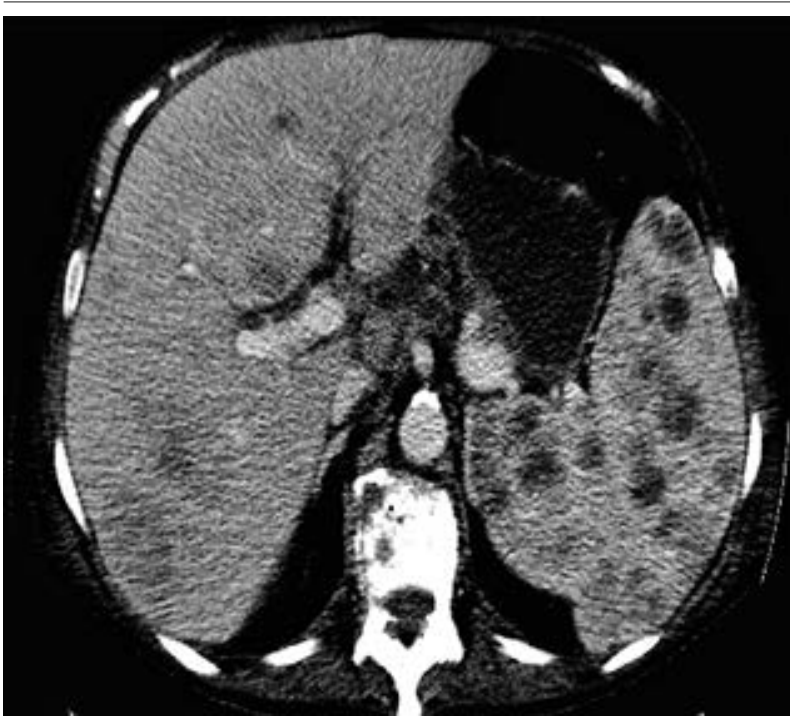

Fig 4. Abdominal CT scan (parenchymal phase) revealing multiple liver and splenic lesions, predominantly in the spleen.

\section{Discussion}

Splenic sarcomas are the rarest primary tumors arising in the spleen with only three cases of leiomyosarcoma reported so far [8-10]. Two of these were in young women (49 and 54 years old) while the third was described in an 87 year old male patient. Previous reports have based their diagnosis on histological evaluation after splenectomy, but in our case the intervention was considered to have an increased risk and biopsy from metastases was elected. Two of the previously reported cases described hemorrhage secondary to the splenic lesions; in our case, the patient's anemia was corrected by blood transfusions and ultrasonography and CT evaluation did not reveal signs of bleeding. Another important aspect in our case is the extent of the disease; the previously reported cases had only splenic involvement, while our patient presented liver, bones and lymph nodes metastases.
Performing CEUS in our patient was an important step in assessing the hepatic and splenic lesions, leading to the diagnosis of primary tumor of the spleen. CEUS proved the cystic appearance of the splenic lesions and suggests for the first time the spleen as origin of the malignancy. However, extensive immunohistochemical analysis of the lesions was required for a positive diagnosis.

\section{References}

1. Fotiadis CI, Georgopoulos I, Stoidis C, Patapis P. Primary tumors of the spleen. Int J Biomed Sci 2009;5:85-91.

2. Spier CM, Kjeldsberg CR, Eyre HJ, Behm FG. Malignant lymphoma with primary presentation in the spleen. A study of 20 patients. Arch Pathol Lab Med 1985;109:1076-1080.

3. Iliescu L, Mercan-Stanciu A, Ioanitescu ES, Toma L. Hepatitis C-Associated B-cell Non-Hodgkin Lymphoma: A Pictorial Review. Ultrasound Q 2018;34:156-166.

4. Ioanitescu ES, Copaci I, Mindrut E, et al. Various aspects of Contrast-enhanced Ultrasonography in splenic lesions - a pictorial essay. Med Ultrason 2020;22:2521.

5. Sidhu PS, Cantisani V, Dietrich CF, et al. The EFSUMB Guidelines and Recommendations for the Clinical Practice of Contrast-Enhanced Ultrasound (CEUS) in Non-Hepatic Applications: Update 2017 (Long Version). Ultraschall Med 2018;39:e2-e44.

6. Trenker C, Görg C, Freeman S, et al. WFUMB Position Paper-Incidental Findings, How to Manage: Spleen. Ultrasound Med Biol 2021;47:2017-2032.

7. Omar A, Freeman S. Contrast-enhanced ultrasound of the spleen. Ultrasound $2016 \mathrm{Feb} ; 24: 41-49$.

8. Farah BL, Chee Y, Ching S, Tan C. Human Pathology: Case Reports. Primary splenic leiomyosarcoma as an exceptionally rare cause of ruptured splenomegaly - A case report and review of primary splenic sarcomas. Hum Pathol Case Reports 2020;22:200452.

9. Piovanello P, Viola V, Costa G, et al. Locally advanced leiomyosarcoma of the spleen. A case report and review of the literature. World J Surg Oncol 2007;5:135.

10. Daudia AT, Walker S, Morgan B, Lloyd DM. Images in surgery Leiomyosarcoma of the spleen. Surgery 2001;130:893-894. 